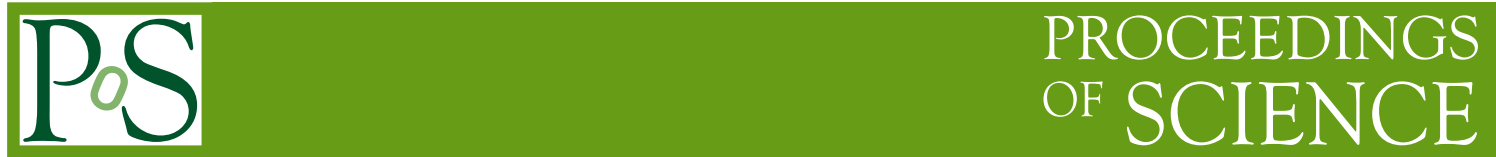

\title{
Renormalization of Flavor Singlet and Nonsinglet Fermion Bilinear Operators
}

\author{
Martha Constantinou $^{a}$, Marios Hadjiantonis ${ }^{a, b}$, Haralambos Panagopoulos ${ }^{* a}$ \\ a Department of Physics, University of Cyprus, Nicosia CY-1678, Cyprus \\ ${ }^{b}$ Present address: Department of Physics, University of Michigan, Ann Arbor, MI 48109, USA \\ E-mail: constantinou . martha@ucy - ac.cy, mchatz12@ucy • ac.cy, \\ haris@ucy.ac.cy
}

We compute the difference in the renormalization of flavor singlet and nonsinglet fermion bilinear operators, to two loops in perturbation theory. Our results are applicable to a rather wide class of lattice actions with Symanzik improved gluons, stout links and clover fermions, including the Twisted Mass and SLiNC actions.

A more detailed presentation of our results, along with relevant references, will appear in our forthcoming publication [1].

The 32nd International Symposium on Lattice Field Theory,

23-28 June, 2014

Columbia University New York, NY

\footnotetext{
* Speaker.
} 


\section{Introduction}

In this work we study the renormalization of fermion bilinears $\mathscr{O}_{\Gamma}=\bar{\psi} \Gamma \psi$ on the lattice, where $\Gamma=\hat{1}, \gamma_{5}, \gamma_{\mu}, \gamma_{5} \gamma_{\mu}, \gamma_{5} \sigma_{\mu \nu}$. We consider flavor singlet $\left(\sum_{f} \bar{\psi}_{f} \Gamma \psi_{f}\right.$, f: flavor index $)$ as well as nonsinglet operators, to two loops in perturbation theory. Our calculations were performed making use of a large family of lattice actions, including Symanzik improved gluons, Wilson fermions with stout links, and clover fermions; twisted mass actions (with Iwasaki or tree level Symanzik gluons) and the SLiNC action are members of this family.

The most demanding parts of this study are the computation of the 2-point Green's functions of $\mathscr{O}_{\Gamma}$, up to two loops and, as a prerequisite, the two-loop fermion self-energy. From these Green's functions we extract the renormalization functions for the fermion field and for $\mathscr{O}_{\Gamma}: Z_{\psi}^{L, Y}, Z_{\Gamma}^{L, Y}(L$ : lattice regularization, $Y\left(=R I^{\prime}, \overline{M S}\right)$ : renormalization schemes). As a check on our results, we have computed them in an arbitrary covariant gauge. Our expressions for $Z_{\psi}^{L, Y}, Z_{\Gamma}^{L, Y}$ can be generalized, in a straightforward manner, to fermionic fields in an arbitrary representation.

Flavor singlet operators are relevant for a number of hadronic properties including, e.g., topological features or the spin structure of hadrons. Matrix elements of such operators are notoriously difficult to study via numerical simulations, due to the presence of (fermion line) disconnected diagrams, which in principle require evaluation of the full fermion propagator. In recent years there has been some progress in the numerical study of flavor singlet operators; furthermore, for some of them, a nonperturbative estimate of their renormalization has been obtained using the FeynmanHellmann relation [2]. Perturbation theory can give an important cross check for these estimates, and provide results for operators which are more difficult to renormalize nonperturbatively.

Given that for the renormalization of flavor nonsinglet operators one can obtain quite accurate nonperturbative estimates, we will focus on the perturbative evaluation of the difference between the flavor singlet and nonsinglet renormalization; this difference first shows up at two loops.

Perturbative computations beyond one loop for Green's functions with nonzero external momenta are technically quite involved, and their complication is greatly increased when improved gluon and fermion actions are employed. For fermion bilinear operators, the only two-loop computations in standard perturbation theory thus far have been performed by our group [3], employing Wilson gluons and Wilson/clover fermions. Similar investigations have been carried out in the context of stochastic perturbation theory [4].

Further composite fermion operators of interest, to which one can apply our perturbative techniques, are higher dimension bilinears such as: $\bar{\psi} \Gamma D^{\mu} \psi$ (appearing in hadron structure functions) and four-fermion operators such as: $\left(\bar{s} \Gamma_{1} d\right)\left(\bar{s} \Gamma_{2} d\right)$ (appearing in $\Delta S=2$ transitions, etc.); in these cases, complications such as operator mixing greatly hinder nonperturbative methods of renormalization, making a perturbative approach all that more essential.

\section{Definitions and Calculational Setup}

In standard notation, the action consists of a gluon part, $S_{G}$, and a fermion part, $S_{W}+S_{S W}$ :

$$
S_{W}=-\frac{a_{\mathrm{L}}^{3}}{2} \sum_{n, \mu}\left[\bar{\Psi}(\vec{n})\left(\left(r-\gamma_{\mu}\right) \tilde{U}_{\vec{n}, \vec{n}+\hat{\mu}} \Psi(\vec{n}+\hat{\mu})+\left(r+\gamma_{\mu}\right) \tilde{U}_{\vec{n}-\hat{\mu}, \vec{n}}^{\dagger} \Psi(\vec{n}-\hat{\mu})-2 r \Psi(\vec{n})\right)\right](
$$




$$
\begin{array}{r}
S_{S W}=-a_{\mathrm{L}}^{5} \sum_{n} c_{S W} \bar{\Psi}(\vec{n}) \frac{1}{4} \sigma_{\mu \nu} \hat{G}_{\mu v}(\vec{n}) \Psi(\vec{n}), \quad \hat{G}_{\mu v}(\vec{n})=\left[Q_{\mu v}(\vec{n})-Q_{v \mu}(\vec{n})\right] /\left(8 a_{\mathrm{L}}^{2}\right), \\
Q_{\mu \nu}=\quad \begin{array}{l}
U_{\vec{n}, \vec{n}+\hat{\mu}} U_{\vec{n}+\hat{\mu}, \vec{n}+\hat{\mu}+\hat{v}} U_{\vec{n}+\hat{\mu}+\hat{v}, \vec{n}+\hat{v}} U_{\vec{n}+\hat{v}, \vec{n}}+U_{\vec{n}, \vec{n}+\hat{v}} U_{\vec{n}+\hat{v}, \vec{n}+\hat{v}-\hat{\mu}} U_{\vec{n}+\hat{v}-\hat{\mu}, \vec{n}-\hat{\mu}} U_{\vec{n}-\hat{\mu}, \vec{n}} \\
+U_{\vec{n}, \vec{n}-\hat{\mu}} U_{\vec{n}-\hat{\mu}, \vec{n}-\hat{\mu}-\hat{v}} U_{\vec{n}-\hat{\mu}-\hat{v}, \vec{n}-\hat{v}} U_{\vec{n}-\hat{v}, \vec{n}}+U_{\vec{n}, \vec{n}-\hat{v}} U_{\vec{n}-\hat{v}, \vec{n}-\hat{v}+\hat{\mu}} U_{\vec{n}-\hat{v}+\hat{\mu}, \vec{n}+\hat{\mu}} U_{\vec{n}+\hat{\mu}, \vec{n}}
\end{array}
\end{array}
$$

The fermion action may also contain standard and twisted mass terms, but they only contribute beyond two loops to the difference between flavor singlet and nonsinglet renormalizations; this is true in mass-independent schemes, such as $R I^{\prime}$ and $\overline{M S}$, in which renormalized masses vanish. The quantities $\tilde{U}_{\vec{n}, \vec{n}+\hat{\mu}}$, appearing above, are stout links, defined as: $\tilde{U}_{\vec{n}, \vec{n}+\hat{\mu}}=e^{i Q_{\hat{\mu}}(\vec{n})} U_{\vec{n}, \vec{n}+\hat{\mu}}$, where:

$$
Q_{\hat{\mu}}(\vec{n})=\frac{\omega}{2 i}\left[V_{\hat{\mu}}(\vec{n}) U_{\vec{n}, \vec{n}+\hat{\mu}}^{\dagger}-U_{\vec{n}, \vec{n}+\hat{\mu}} V_{\hat{\mu}}^{\dagger}(\vec{n})-\frac{1}{N_{c}} \operatorname{Tr}\left(V_{\hat{\mu}}(\vec{n}) U_{\vec{n}, \vec{n}+\hat{\mu}}^{\dagger}-U_{\vec{n}, \vec{n}+\hat{\mu}} V_{\hat{\mu}}^{\dagger}(\vec{n})\right)\right]
$$

and $V_{\hat{\mu}}(\vec{n})$ is the sum of 6 staples joining sites $\vec{n}$ and $\vec{n}+\hat{\mu}$. Both the stout coefficient $\omega$ and the clover coefficient $c_{S W}$ will be treated as free parameters, for wider applicability of the results.

We employ a Symanzik improved gluon action, of the form:

$$
S_{G}=\frac{2}{g^{2}} \operatorname{Re} \operatorname{Tr}\left[c_{0} \sum_{\text {Plaq }}\left(1-U_{\text {Plaq }}\right)+c_{1} \sum_{\text {Rect }}\left(1-U_{\text {Rect }}\right)+c_{2} \sum_{\text {Chair }}\left(1-U_{\text {Chair }}\right)+c_{3} \sum_{\text {Paral }}\left(1-U_{\text {Paral }}\right)\right]
$$

where: $U_{\text {Plaq }}\left(U_{\text {Rect }}, U_{\text {Chair }}, U_{\text {Paral }}\right)$ is the product of links around a $1 \times 1$ plaquette (the three independent 6-link Wilson loops: " $2 \times 1$ rectangle", "chair", "parallelogram"), and the Symanzik coefficients $c_{i}$ satisfy: $c_{0}+8 c_{1}+16 c_{2}+8 c_{3}=1$. The algebraic part of our computation was carried out for generic values of $c_{i}$; for the numerical integration over loop momenta we selected a number of commonly used sets of values, some of which are shown in Table 1.

\begin{tabular}{ccccc}
\hline \hline Action & $c_{0}$ & $c_{1}$ & $c_{2}$ & $c_{3}$ \\
\hline Wilson & 1 & 0 & 0 & 0 \\
Tree-Level Symanzik & $5 / 3$ & $-1 / 12$ & 0 & 0 \\
Iwasaki & 3.648 & -0.331 & 0 & 0 \\
DBW2 & 12.2688 & -1.4086 & 0 & 0 \\
\hline \hline
\end{tabular}

Table 1: Selected sets of values for Symanzik coefficients

Denoting all bare quantities in the Lagrangian with the subscript "o", the corresponding renormalized quantities and renormalization functions read:

$$
A_{\mu \circ}^{a}=\sqrt{Z_{A}} A_{\mu}^{a}, \quad c_{\circ}^{a}=\sqrt{Z_{c}} c^{a}, \quad \psi_{\circ}=\sqrt{Z_{\psi}} \psi, \quad g_{\circ}=\mu^{\varepsilon} Z_{g} g, \quad \alpha_{\circ}=Z_{\alpha}^{-1} Z_{A} \alpha
$$

(respectively: gauge field, ghost field, fermion field, coupling constant, gauge parameter) where $\mu$ is the mass scale introduced to ensure that $g_{\circ}$ has the correct dimensionality in $d=4-2 \varepsilon$ dimensions. $Z_{A}, Z_{c}, Z_{g}, Z_{\alpha}$ are only needed to one loop, and their values are known.

Aside from $c_{S W}$ and $\omega$, we treat as free parameters: $g_{0}, \alpha, N_{f}\left(N_{c}\right)$ : number of flavors (colors). 
The 2-point amputated Green's functions of the operators $\mathscr{O}_{\Gamma}$ can be written in the form:

$$
\begin{aligned}
\Sigma_{S}\left(q a_{\mathrm{L}}\right) & =\hat{1} \Sigma_{S}^{(1)}\left(q a_{\mathrm{L}}\right) \\
\Sigma_{P}\left(q a_{\mathrm{L}}\right) & =\gamma_{5} \Sigma_{P}^{(1)}\left(q a_{\mathrm{L}}\right) \\
\Sigma_{V}\left(q a_{\mathrm{L}}\right) & =\gamma_{\mu} \Sigma_{V}^{(1)}\left(q a_{\mathrm{L}}\right)+q^{\mu}\left(q / q^{2}\right) \Sigma_{V}^{(2)}\left(q a_{\mathrm{L}}\right) \\
\Sigma_{A V}\left(q a_{\mathrm{L}}\right) & =\gamma_{5} \gamma_{\mu} \Sigma_{A V}^{(1)}\left(q a_{\mathrm{L}}\right)+\gamma_{5} q^{\mu}\left(q / q^{2}\right) \Sigma_{A V}^{(2)}\left(q a_{\mathrm{L}}\right) \\
\Sigma_{T}\left(q a_{\mathrm{L}}\right) & =\gamma_{5} \sigma_{\mu v} \Sigma_{T}^{(1)}\left(q a_{\mathrm{L}}\right)+\gamma_{5}\left(q / q^{2}\right)\left(\gamma_{\mu} q_{v}-\gamma_{v} q_{\mu}\right) \Sigma_{T}^{(2)}\left(q a_{\mathrm{L}}\right)
\end{aligned}
$$

$\left(S, P, V, A V, T\right.$ correspond to: $\left.\Gamma=\hat{1}, \gamma_{5}, \gamma_{\mu}, \gamma_{5} \gamma_{\mu}, \gamma_{5} \sigma_{\mu \nu} . \Sigma_{\Gamma}^{(1)}=1+\mathscr{O}\left(g_{\circ}^{2}\right), \Sigma_{\Gamma}^{(2)}=\mathscr{O}\left(g_{\circ}^{2}\right)\right)$.

The $R I^{\prime}$ renormalization scheme is defined by imposing renormalization conditions on matrix elements at a scale $\bar{\mu}$, where (just as in $\overline{M S}$ ): $\bar{\mu}=\mu\left(4 \pi / e^{\gamma_{\mathrm{E}}}\right)^{1 / 2}$. For the renormalized operators: $\mathscr{O}_{\Gamma}^{R I^{\prime}}=Z_{\Gamma}^{L, R I^{\prime}}\left(a_{\mathrm{L}} \bar{\mu}\right) \mathscr{O}_{\Gamma \circ}\left(\mathscr{O}_{\Gamma \circ}=\bar{\psi} \Gamma \psi\right)$, one defines $Z_{\Gamma}^{L, R I^{\prime}}$ via:

$$
\lim _{a_{\mathrm{L}} \rightarrow 0}\left[Z_{\psi}^{L, R I^{\prime}} Z_{\Gamma}^{L, R I^{\prime}} \Sigma_{\Gamma}^{(1)}\left(q a_{\mathrm{L}}\right)\right]_{q^{2}=\bar{\mu}^{2}}=1
$$

This renormalization prescription does not involve $\Sigma_{\Gamma}^{(2)}$; nevertheless, renormalizability of the theory implies that $Z_{\Gamma}^{L, R I^{\prime}}$ will render the entire Green's function finite. An alternative prescription, more appropriate for nonpertubative renormalization is:

$$
\lim _{a_{\mathrm{L}} \rightarrow 0}\left[Z_{\psi}^{L, R I^{\prime}} Z_{\Gamma}^{L, R I^{\prime}} \operatorname{tr}\left(\Gamma \Sigma_{\Gamma}\left(q a_{\mathrm{L}}\right)\right) / \operatorname{tr}(\Gamma \Gamma)\right]_{q^{2}=\bar{\mu}^{2}}=1
$$

The two prescriptions differ between themselves (for $\mathrm{V}, \mathrm{AV}, \mathrm{T}$ ) by a finite amount which can be deduced from lower loop calculations combined with continuum results.

Conversion of renormalization functions from $R I^{\prime}$ to the $\overline{M S}$ scheme is facilitated by the fact that renormalized Green's functions are regularization independent; thus the finite conversion factors $(D R \equiv$ Dimensional Regularization):

$$
C_{\Gamma}(g, \alpha) \equiv \frac{Z_{\Gamma}^{L, R I^{\prime}}}{Z_{\Gamma}^{L, \overline{M S}}}=\frac{Z_{\Gamma}^{D R, R I^{\prime}}}{Z_{\Gamma}^{D R, \overline{M S}}}
$$

can be evaluated in DR, leading to: $Z_{\Gamma}^{L, \overline{M S}}=Z_{\Gamma}^{L, R I^{\prime}} / C_{\Gamma}(g, \alpha)$. For the pseudoscalar and axial vector operators, in order to satisfy Ward identities, additional finite factors $Z_{5}^{P}(g)$ and $Z_{5}^{A V}(g)$, calculable in $\mathrm{DR}$, are required:

$$
Z_{P}^{L, \overline{M S}}=Z_{P}^{L, R I^{\prime}} /\left(C_{S} Z_{5}^{P}\right), \quad Z_{A V}^{L, \overline{M S}}=Z_{A V}^{L, R I^{\prime}} /\left(C_{V} Z_{5}^{A V}\right)
$$

These factors are gauge invariant; we also note that the value of $Z_{5}^{A V}$ for the flavor singlet operator differs from that of the nonsinglet one.

\section{Computation and Results}

In the previous Section, the calculational setup was presented in rather general terms. Here we focus on the two-loop difference between flavor singlet and nonsinglet operator renormalization. 
Given that this difference first arises at two loops, we only need the tree level values of: $Z_{\psi}, Z_{A}, Z_{c}$, $Z_{\alpha}, Z_{g}$ and of the conversion factors $C_{\Gamma}, Z_{5}^{P}$ and $Z_{5}^{A V}$. Since the tree level value of $C_{\Gamma}$ equals 1 , the difference up to two loops will not depend on the renormalization scheme.

There are 4 two-loop Feynman diagrams contributing to the above difference in the evaluation of the Green's functions (2.7-2.11), shown in Fig. 1. They all contain an operator insertion inside a closed fermion loop, and therefore vanish in the flavor nonsinglet case.
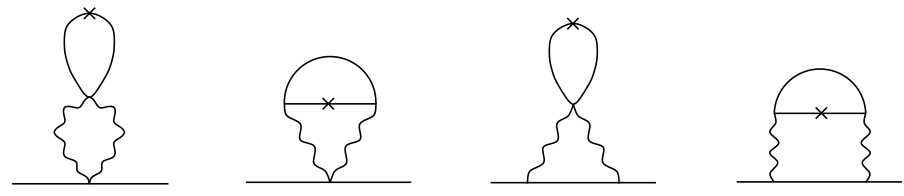

Figure 1: Diagrams contributing to the difference between flavor singlet and nonsinglet values of $Z_{\Gamma}$. Solid (wavy) lines represent fermions (gluons). A cross denotes insertion of the operator $\sum \bar{\psi} \Gamma \psi$.

The above diagrams, evaluated individually, may be IR divergent, due to the presence of two gluon propagators with the same momentum. Comparing to our previous evaluation of these diagrams with Wilson gluons and clover fermions, we will find no new superficial divergences, i.e. no new $\ln ^{2}\left(\bar{\mu} a_{\mathrm{L}}\right)$ terms. However, the presence of stout links and Symanzik gluons leads to considerably longer expressions for the vertices. Also, the gluon propagator must now be inverted numerically for each value of the loop momentum 4-vector (an inversion in closed form exists, but it is not efficient).

The contribution of these diagrams to $Z_{P}, Z_{V}, Z_{T}$ vanishes identically. Only $Z_{S}$ and $Z_{A V}$ are affected. For the Scalar operator, our result can be written in the following form:

$$
\begin{aligned}
Z_{S}^{\text {singlet }}\left(\bar{\mu} a_{\mathrm{L}}\right)- & Z_{S}^{\text {non-singlet }}\left(\bar{\mu} a_{\mathrm{L}}\right)=-\frac{g_{0}^{4}}{(4 \pi)^{4}} c_{F} N_{f}\left[\left(s_{00}+s_{01} \mathbf{c}_{S W}+s_{02} \mathbf{c}_{S W}^{2}+s_{03} \mathbf{c}_{S W}^{3}+s_{04} \mathbf{c}_{S W}^{4}\right)\right. \\
& +\left(s_{10}+s_{11} \mathbf{c}_{S W}+s_{12} \mathbf{c}_{S W}^{2}+s_{13} \mathbf{c}_{S W}^{3}\right) \omega+\left(s_{20}+s_{21} \mathbf{c}_{S W}+s_{22} \mathbf{c}_{S W}^{2}\right) \omega^{2} \\
& \left.+\left(s_{30}+s_{31} \mathbf{c}_{S W}\right) \omega^{3}+s_{40} \omega^{4}\right]+\mathscr{O}\left(g_{0}^{6}\right)
\end{aligned}
$$

$\left(c_{F} \equiv\left(N_{c}^{2}-1\right) /\left(2 N_{c}\right)\right)$. The numerical constants $s_{i, j}$ have been computed for various sets of values of the Symanzik coefficients; their values are listed in Table 2. The computation was performed in a general covariant gauge, confirming that the result is gauge independent, as it should be in $\overline{M S}$. We note from Eq. (3.1) that even single logarithms are absent; thus the result is scale independent.

For the Axial Vector operator we find:

$$
\begin{aligned}
Z_{A V}^{\text {singlet }}\left(\bar{\mu} a_{\mathrm{L}}\right)- & Z_{A V}^{\text {non-singlet }}\left(\bar{\mu} a_{\mathrm{L}}\right)=-\frac{g_{0}^{4}}{(4 \pi)^{4}} c_{F} N_{f}\left[\left(a_{00}+a_{01} \mathbf{c}_{S W}+a_{02} \mathbf{c}_{S W}^{2}+a_{03} \mathbf{c}_{S W}^{3}+a_{04} \mathbf{c}_{S W}^{4}\right)\right. \\
& +\left(a_{10}+a_{11} \mathbf{c}_{S W}+a_{12} \mathbf{c}_{S W}^{2}+a_{13} \mathbf{c}_{S W}^{3}\right) \omega+\left(a_{20}+a_{21} \mathbf{c}_{S W}+a_{22} \mathbf{c}_{S W}^{2}\right) \omega^{2} \\
& \left.+\left(a_{30}+a_{31} \mathbf{c}_{S W}\right) \omega^{3}+a_{40} \omega^{4}+6 \ln \left(\bar{\mu}^{2} a_{\mathrm{L}}^{2}\right)\right]+\mathscr{O}\left(g_{0}^{6}\right)
\end{aligned}
$$

By analogy with the scalar case, the computation was performed in a general gauge and the numerical constants are tabulated in Table 2. We note that the result for the Axial Vector operator has a scale dependence; this is related to the axial anomaly. Finally, the presence of a term of the 


\begin{tabular}{|l|ccc||c|ccc|}
\hline & Wilson & TL Symanzik & Iwasaki & & Wilson & TL Symanzik & Iwasaki \\
\hline$s_{00}$ & $107.76(2)$ & $76.29(1)$ & $42.973(7)$ & $a_{00}$ & $2.051(2)$ & $3.098(3)$ & $5.226(4)$ \\
$s_{01}$ & $-82.27(1)$ & $-69.01(1)$ & $-49.356(8)$ & $a_{01}$ & $-15.033(3)$ & $-12.851(3)$ & $-9.426(3)$ \\
$s_{02}$ & $29.730(2)$ & $26.178(1)$ & $20.312(3)$ & $a_{02}$ & $-5.013(2)$ & $-3.361(1)$ & $-1.3526(7)$ \\
$s_{03}$ & $-3.4399(7)$ & $-2.9533(5)$ & $-2.2166(3)$ & $a_{03}$ & $2.1103(3)$ & $1.7260(1)$ & $1.1251(2)$ \\
$s_{04}$ & $-2.2750(4)$ & $-1.6403(3)$ & $-0.8547(2)$ & $a_{04}$ & $0.0434(2)$ & $0.01636(1)$ & $-0.01074(5)$ \\
$s_{10}$ & $-1854.4(2)$ & $-1107.0(1)$ & $-444.69(4)$ & $a_{10}$ & $43.75(1)$ & $36.66(1)$ & $25.827(9)$ \\
$s_{11}$ & $506.26(5)$ & $364.01(3)$ & $192.35(1)$ & $a_{11}$ & $76.993(3)$ & $57.190(3)$ & $31.768(2)$ \\
$s_{12}$ & $-95.42(2)$ & $-70.94(1)$ & $-40.162(6)$ & $a_{12}$ & $44.260(4)$ & $29.363(2)$ & $12.962(1)$ \\
$s_{13}$ & $7.494(1)$ & $5.356(1)$ & $2.8030(4)$ & $a_{13}$ & $-4.4660(6)$ & $-3.3740(5)$ & $-1.8710(2)$ \\
$s_{20}$ & $18317(2)$ & $10081(1)$ & $3511.3(4)$ & $a_{20}$ & $-126.45(1)$ & $-92.853(7)$ & $-50.378(1)$ \\
$s_{21}$ & $-2061.8(2)$ & $-1350.7(1)$ & $-595.79(7)$ & $a_{21}$ & $-259.59(3)$ & $-175.65(2)$ & $-81.45(1)$ \\
$s_{22}$ & $202.75(7)$ & $133.19(4)$ & $59.25(2)$ & $a_{22}$ & $-107.48(1)$ & $-67.737(8)$ & $-27.500(3)$ \\
$s_{30}$ & $-96390(10)$ & $-50300(5)$ & $-16185(2)$ & $a_{30}$ & $295.76(3)$ & $198.78(2)$ & $90.96(1)$ \\
$s_{31}$ & $3784.8(4)$ & $2336.0(3)$ & $925.6(1)$ & $a_{31}$ & $400.05(5)$ & $253.87(3)$ & $104.74(1)$ \\
$s_{40}$ & $213470(20)$ & $106940(10)$ & $32572(3)$ & $a_{40}$ & $-348.41(4)$ & $-220.12(3)$ & $-90.11(1)$ \\
\hline
\end{tabular}

Table 2: Numerical coefficients for the Scalar and Axial Vector operators. The errors quoted stem from numerical integration over loop momenta.

form $\gamma_{5} q^{\mu} q / q^{2}$ in the Green's function (2.10) implies that, in the alternative $R I^{\prime}$ scheme mentioned in Section 2, one must add $\left(g_{0}^{4} /(4 \pi)^{4}\right) c_{F} N_{f}$ to the above result.

In Fig. 2 we illustrate our results by selecting parameter values appropriate to the ETMC action with Iwasaki gluons, $N_{f}=2+1+1, \beta=2 N_{c} / g_{\circ}^{2}=1.95, \bar{\mu}=1 / a_{\mathrm{L}}$ and standard/stout links for the Wilson part of the fermion action. Similarly, Fig. 3 presents our results for parameter values appropriate to the SLiNC action, with Tree-Level Symanzik gluons, $N_{f}=3, \beta=2 N_{c} c_{0} / g_{\circ}^{2}=5.5$, $\bar{\mu}=1 / a_{\mathrm{L}}$.

Further extensions of the present work include the application to other actions currently used in numerical simulations, including actions with more steps of stout smearing. In these cases, additional contributions to the renormalization functions are more convergent, and thus their perturbative treatment is simpler; nevertheless, the sheer size of the vertices $\left(\sim 10^{6}\right.$ terms for two stout-smearing steps) renders the computation quite cumbersome. Another possible extension of this work regards several variants of staggered fermion actions. Finally, extended versions of $\bar{\psi} \Gamma \psi$ may be studied; in this case another four Feynman diagrams must be added to those of Fig. 1, leading to longer but convergent loop integrands.

Acknowledgments: Work supported by the Cyprus Research Promotion Foundation under Con-

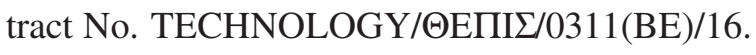

\section{References}

[1] M. Constantinou, M. Hadjiantonis and H. Panagopoulos, Renormalization of Flavor Singlet Fermion Operators with Improved Actions, in preparation. 

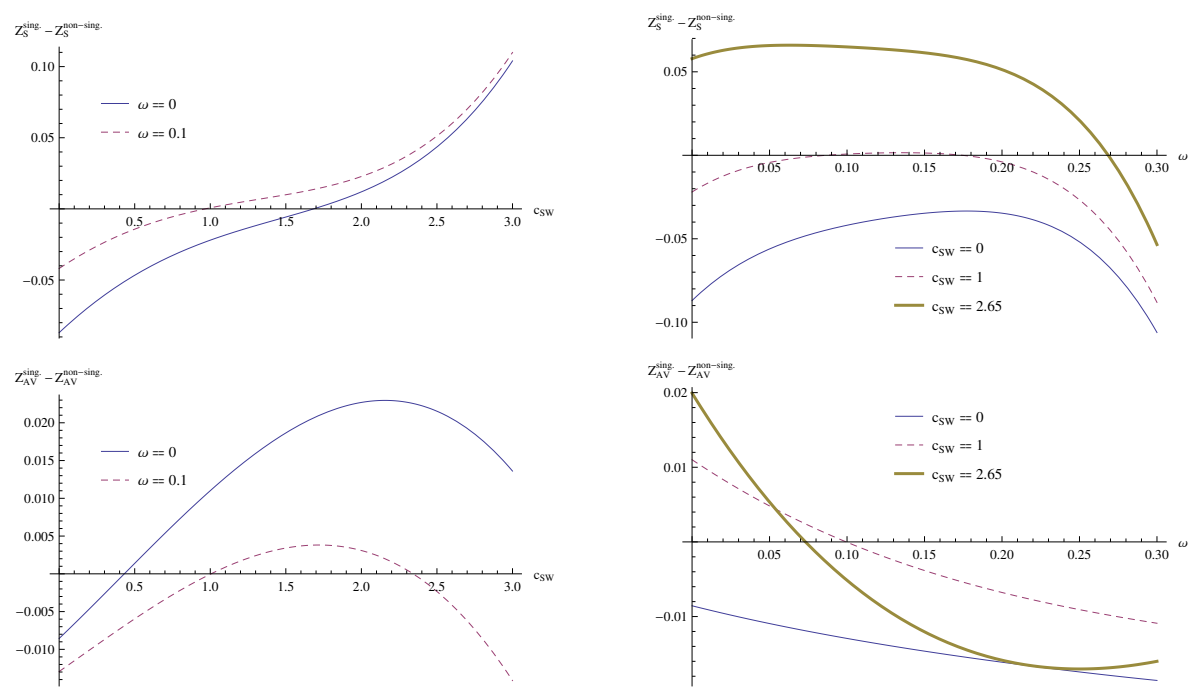

Figure 2: Plots of $Z_{\Gamma}^{\text {singlet }}-Z_{\Gamma}^{\text {nonsiglet }}$, for $\Gamma=S$ (top panels) and $\Gamma=A V$ (bottom), as a function of $c_{S W}$ (left) and $\omega$ (right). Parameter values relevant for ETMC action ( $N_{f}=4$, Iwasaki gluons, $\left.\beta=1.95\right)$.
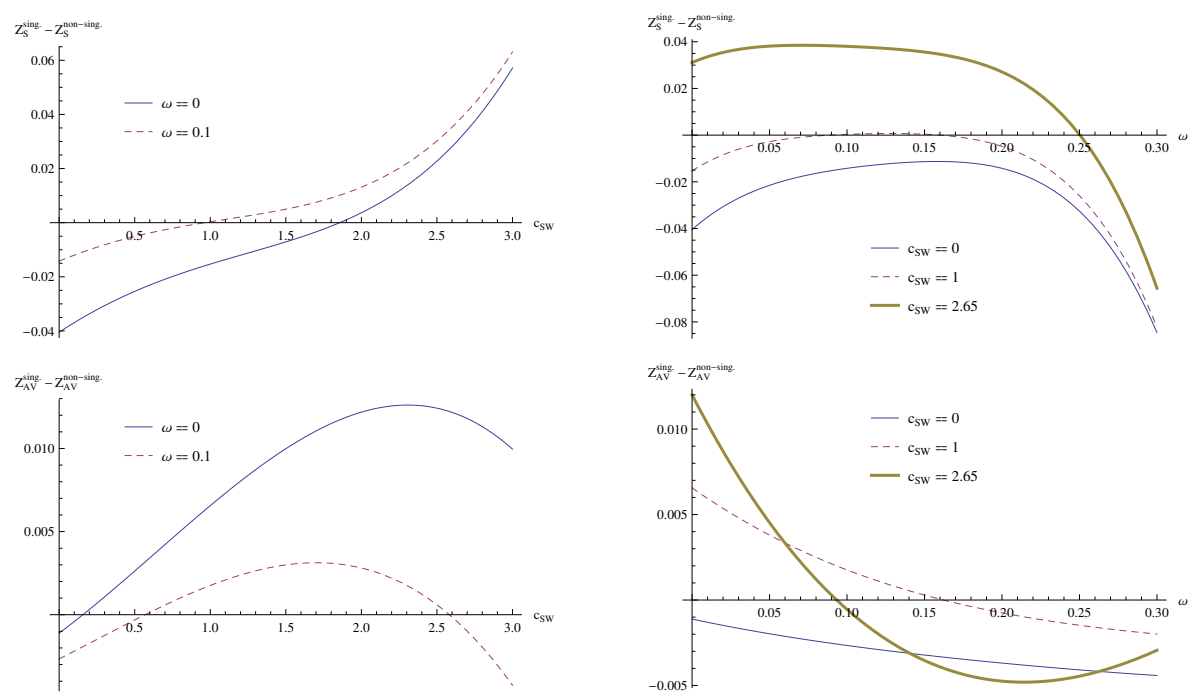

Figure 3: Plots of $Z_{\Gamma}^{\text {singlet }}-Z_{\Gamma}^{\text {nonsiglet }}$, for $\Gamma=S$ (top panels) and $\Gamma=A V$ (bottom), as a function of $c_{S W}$ (left) and $\omega$ (right). Parameter values relevant for SLiNC action $\left(N_{f}=3\right.$, Tree level Symanzik gluons, $\left.\beta=5.5\right)$.

[2] A. J. Chambers, R. Horsley, Y. Nakamura et al., A novel approach to nonperturbative renormalization of singlet and nonsinglet lattice operators, arXiv:1410.3078.

[3] A. Skouroupathis, H. Panagopoulos, Two-loop renormalization of scalar and pseudoscalar fermion bilinears on the lattice, PRD 76 (2007) 094514 [arXiv: 0707.2906 ]; Two-loop renormalization of vector, axial-vector and tensor fermion bilinears on the lattice, PRD 79 (2009) 094508 [arXiv:0811.4264].

[4] M. Brambilla, F. Di Renzo, M. Hasegawa, High-loop perturbative renormalization constants for Lattice QCD, arXiv:1402.6581. 